\title{
THE SKIN OF THE HAND
}

\section{The Immediate Treatment of Crush Injuries and Lacerations}

\author{
By JoHn N. Barron, F.R.C.S.(Ed.)
}

\section{Introduction}

The importance of the surgery of the hand has been unfortunately under-estimated in the past. This lack of systematic teaching on the subject is reflected in general practice and the results of such treatment provide a distressingly large number of patients who require lengthy reconstructive procedures.

Since the incidence of injury falls most heavily upon the fit and able worker, it is obviously most important to concentrate attention on this vital problem.

\section{Principles of Treatment}

A thorough knowledge of the minute anatomy and the function of the hand is the only basis for correct diagnosis and treatment. Much of hand surgery is the surgery of millimetres, and the operator should be prepared to equip himself to this degree of intimacy with its structure and its function.

\section{Atraumatic Technique}

The response of the tissues to a crushing injury is oedema and fibrosis, degeneration and cicatrix. This is just as true of the localized crush of a haemostat as it is of a power-press injury and varies only in degree. Multiple small crushing traumata at operation may cause a widespread vascular protest, with its inevitable sequence of oedema and fibrous replacement.

So narrow is the margin of play between a tendon and its sheath and so intimate the structure of a capsule and its ligaments, that even a minor invasion by the fibroblast may impair dexterity and function. It is well, then, at operation to limit the necessary trauma so that normal structures are not reflexly injured. The lightest touch, a tourniquet and the finest instruments are essential for this work. Sharp dissection with sharp instruments and the handling of tissues with fine hooks instead of forceps will minimize reaction and encourage scar-free healing.

A bloodless field is essential for accurate surgery and a pneumatic tourniquet can be left in position for an hour with safety. If on removal of the pressure cuff, the operation area is compressed and the arm elevated for two or three minutes most of the capillary oozing will have ceased and the remaining bleeding points can be tied with a $6 / 0$ catgut. Absolute haemostasis should be the rule as bleeding will persist in places unsupported by the dressing such as the thenar space and interdigital webs. Deep haematomata cause considerable disturbance and lead to massive fibrosis.

Most surgical procedures on the hand should be followed by a carefully applied pressure dressing. Wool should be packed in against all the skin surfaces and built up until it can be evenly compressed by an elastic bandage so that all parts are supported, oedema is prevented and the circulation sustained. Fortyeight hours elevation aids venous and lymphatic drainage, minimizes post-operative discomfort and swelling and allows an early return of function.

\section{The Pathology of Injury}

\section{Cutting injuries}

Apart from acute infections, hand injuries are caused by cutting, crushing and burning. It is important always to consider the differences between a 'crush' and a 'cut.' A laceration made by a sharp knife or a razor damages the minimum number of cells compatible with the size of the injury. Immediate drainage on to the wound surface is automatically provided for the damaged tissues, and intra vascular clotting remains localized to the capillaries surrounding the wound. The reaction to injury then tends to involve only the cell strata of which the wound is formed so long as infection does not supervene. 


\section{Crushing injuries}

An injury caused by a blunt cutting instrument will not only lacerate but, by virtue of the superadded crushing effect, will damage many more cells in strata further away from the wound surface. These crushed tissues have no drainage to the surface and oedema results. Whether by reflex mechanism 'or by toxic absorption, a state of increased capillary permeability supervenes in the limb, the widespread oedema still further impeding drainage through the veins and lymphatics. Intra-vascular clotting starts distally and the blood supply if still further diminished may result in avascular necrosis of the superficial layers of the wound. Here is an excellent pabulum in which infection can flourish.

The pure 'crush' injury, although it may not lacerate or fracture, can, by causing severe metabolic disturbances, result in total disability - the frozen hand. The oedematous reaction far from being obviously confined to the sub-cutaneous planes, invades the musculature, the deep spaces, the tendon sheaths and the joints. If it is not energetically treated from the outset, much of the fluid becomes fibrinized and later cicatrises. Muscle, tendon and capsular tissues subjected to high tensions and to anoxia degenerate, and the motor functions of the hand suffer. Digital nerves and their complex sensory ends are strangled in the scar, and sensory and atrophic disturbances are the sequel.

In any acute injury, it is valuable to assess the ratio of the 'crush ' to the ' cut.' Not only in industrial injuries does it lead to much interesting information about the work our patients do, but in all injuries it enables us more accurately to hazard the risk of infection and it points the way to the correct treatment.

\section{Burning injuries}

Injuries by burning can be considered as thermal, electrical and chemical. The thermal burn evokes a very similar reaction to that caused by a crush injury. There is much variation in effect depending upon the temperature and the length of exposure. Here the skin is first destroyed and, for purposes of treatment, it is convenient to divide burns into two groups-partial thickness skin loss and total thickness skin loss. The first group comprise degrees $I$ and 2 in the Dupuytren's classification, and the second group degrees 3 to 6 . A noteworthy point in the pathology of burns is that there is always more tissue loss. than that occasioned by the heat itself. This takes the form of spreading cell necrosis due to added infection, progressive intra-vascular coagulation and degeneration due to oedema and raised tissue tension. This sequence with its crippling effects can, to a large degree, be controlled if rational treatment is instituted at the outset. These are the factors which make the diagnosis of depth of all but the severest burns difficult if not impossible during the first few days.' The best indication is gained from a study of the causation of the burn rather than a clinical examination of the wound.

\section{Electrical burns}

Electrical burns are caused mainly by the heat generated in the tissues due to their resistance to the passage of the current. Two features are worthy of mention, first that the burnt area is well demarcated, and second that the necrosed tissues are dehydrated by the passage of the current. These facts suggest immediate and active surgical treatment and excision and replacement can give results unobtainable by any other method of treatment.

\section{Chemical burns}

Chemical burns provide the greatest puzzle in diagnosis of depth. Much depends upon the strength of the caustic and upon the amount of sweat and dirt on the skin itself and, of course, upon the skin thickness at the site of injury. Generally speaking, pure Lysol and the concentrated strong acids and alkalis will destroy the full skin thickness of the forearm or the dorsum of the hand in from to to 20 seconds, but will take up to a minute to produce a similar injury of the palmar skin of a workman. These burns remain demarcated unless the contact period is long, or they are followed by infection.

\section{The Immediate Treatment of Hand} Injuries

\section{Crush injuries}

These injuries are common and are caused by a wide variety of accidents. In industry, presses and mills are the worst offenders apart from the everyday occurrence of a 
heavy weight dropping on the hand. In the home, serious disabilities may be caused by the slamming of a door, and in this case the fingers are usually involved. ' Crush' injuries are either closed or open. The skin may split in a plane at right angles to the crushing force and the soft tissues may be extruded thence to the exterior. There may or may not be associated fractures. Pain is severe and prolonged and is due to the trauma to the sensory nerve ends. Oedema is soon apparent and may involve the whole hand. The skin in the areas subjected to the greatest force usually shows evidence of bruising and ecchymosis whilst necrosis and sloughing may occur.

\section{Treatment}

Unless there are special indications for the treatment of fractures, the hand should be put up in the position of function. A few degrees of dorsi flexion at the wrist, all finger joints in about $45^{\circ}$ of flexion, the angles increasing a little in succeeding fingers from the index to the fifth. The thumb in palmar abduction and opposition. In this position a firm pressure dressing is applied, and the hand slung to a point overhead by a loop of bandage or strapping. Severe cases, particularly where there are associated fractures, should be chilled with ice bags and the temperature kept between $60^{\circ}$ and $65^{\circ} \mathrm{F}$. This is an efficient anodyne and prevents congestion and oedema. Any case where there are gaping lacerations which cannot be sutured owing to tension should be skin grafted and the graft removed later if the skin is redundant. This prevents a granulating surface from forming, encourages early movements, and decreases the risk of infection.

\section{The crushed finger tip}

Cases of this sort requiring treatment usually present in addition a nail injury or a fractured terminal phalanx. The basic treatment is again the pressure dressing, but the nail or phalanx may need attention. The subungual haematoma should be drained by a drill hole through the nail and unless this is done, necrosis of the nail bed and the phalanx can be expected if the tension is high. If the nail bed is split the nail should be sutured into normal position to prevent a cleft or other irregularities from forming and the drill hole should still be made. If there is partial avulsion of the nail bed from the bone the following technique should be adopted. A small mould is made in Stent wax or plaster of the nail of the same finger on the opposite hand. This is made in the form of a thin plaque, curved to fit the nail exactly. It is then applied to the injured nail and accurately held in position by a pressure dressing. Many gross deformities can be thus prevented. Fracture dislocations of the distal interphalangeal joints should be put up in traction, preferably on a Bohler's splint.

\section{Lacerations without skin loss}

Under good conditions of treatment, all wounds of the hand which can be explored under direct vision, should be closed. In practice, the main exception to this rule is the perforating injury of the palm or fingers. When the hand has been pierced by a bullet or a sharp instrument, it is impracticable to explore all the interstices of the wound and in attempting to do so potential pathways for dissemination of infection are opened up. Skin and subcutaneous tissue edges should be excised and the wound lightly packed. If desired, a fine 35 gauge stainless steel wire suture can be placed across the wound and tied a few days later if infection is not present. These cases should receive prophylactic penicillin administration. If a ' crush' element is present in these injuries and this may be marked if bone is involved, pressure dressing, elevation and cooling should be included in the treatment. An accurate diagnosis as to tendon and nerve function should be made at the outset as the picture may later be complicated by swelling and fibrosis.

All other lacerations should be subjected to débridment under local, regional or general anaesthesia and a tourniquet. The extent of tissue removal depends upon the ratio of ' crush' and 'cut' in the causation of the injury, wider excision of the wound surface being necessary when the 'crush' element is predominant. In deep wounds this is most easily done under a slow-flowing stream of saline in which ragged and torn material may easily be identified. By this method it is also possible to make a more accurate differentiation between damaged and viable tissue on the woand surface itself.

With the volar skin of the manual labourer, it is wise to shave off the cornified skin layer 
for $\frac{1}{4}$ in. around the wound margin. This layer is non-elastic and difficult to suture. It inverts the deeper skin layers and in any case separates after the stitches have been removed, resulting in slow and imperfect healing. This point is worth noting also when making incisions for the drainage of acute infections and wounds so treated heal with noticeable rapidity. Débridment having been completed and haemostasis secured, the wound should be sutured. Subcutaneous tissue should be closed with 6/0 catgut only if it tends to retract and leave a dead space. Skin sutures should be accurate, and exact apposition of the flexion creases should be made as stitching proceeds. Lacerations crossing the flexion creases on the volar aspects of the fingers should be broken up by the method of $\mathrm{Z}$ plasty in order to avoid flexion contractures which are otherwise very likely to occur.

The method of $\mathrm{Z}$ plasty as applied here is as follows :-

If $\mathrm{AB}$ represents the wound crossing a flexion crease $C D$ at $O$, take a point $E$ on $A B$ I cm. distal to $O$ and a point $F$ I cm. proximal to $\mathrm{O}$. At $\mathrm{E}$ construct an angle OEX of $45^{\circ}, \mathrm{X}$ being a point on this line in the same sagittal plane as $\mathrm{F}$. Construct a similar angle at $\mathrm{F}$ prolonging the line to $\mathrm{Y}$, a point in the same sagittal plane as $E$. The lines EX and FY are marked on the skin and incised with a knife. Two flaps are then formed-FEX and EFY on bases FX and $\mathrm{EY}$ respectively. These flaps are undermined in the subcutaneous plane back to their bases, and are transposed so that point $E$ lies on point $Y$, and point $F$ on point $X$. Fine sutures are placed round the flaps and closure takes the form shown in Fig. 5. The following stitch will be found useful for the corner of each flap. The needle takes the skin just to the deep layer in the dermis in the recipient angle, passes through the corner of the flap at the same level and again through the recipient skin. When the 'stitch is tied, the flap advances and lies accurately in position.

The effect of the $\mathrm{Z}$ transposition is to lengthen the suture line between $A$ and $B$ so that contracture does not tend to approximate these two points and so flexion deformity at the crease does not take place.

\section{Lacerations with skin loss}

Conservative treatment of skin losses of the hand is a potent cause of dysfunction. In the time taken by all but the smallest of these wounds to heal, fibrous tissue forms, contracts and shortens, distorting the hand and interfering with its mechanical and sensory functions. The prolonged healing period and the loss of dexterity and power owing to disuse, constitute an important economic problem in connection with employment. Too many manhours are wasted waiting for conservative treatment to produce second-rate results. Healing will eventually take place after much delay with a poor type of epithelium unbuffered by normal dermis, contracted, , painful, disfiguring and disabling.

In general, untreated volar skin losses produce flexion deformities of the wrist and fingers. Skin losses on the dorsum of the hand interfere with flexion at the metacarpophalangeal joints and draw the thumb into adduction. Skin losses on the backs of the fingers produce extension or hyperextension deformities at the interphalangeal joints. Persistent irritation from infection together with the contraction of the wound causes oedema. Even when healed, the presence of the scar not infrequently causes sympathetic vascular disturbances with atrophic changes in digits due to arterial spasm and capillary paralysis. The effects of this syndrome may be widespread in the hand and the changes may cause permanent crippling. It can be seen then that there are strong indications for the active surgical treatment of skin loss and this consists of the early replacement of the skin defect.

\section{Principles of Skin Repair}

It is an axiom of reconstructive surgery that lost tissues should be replaced by similar tissues and the more this is adhered to the more successful will be the repair. It is often impossible to observe this rule strictly but if it is borne in mind the design of the repair will be on sound lines and the outcome assured. There is, therefore, a primary necessity of making a studied diagnosis of the tissue loss and a careful evaluation of the part played by each of the missing structures.

\section{Subcutaneous tissue}

Thus on the volar surface of the fingers the 

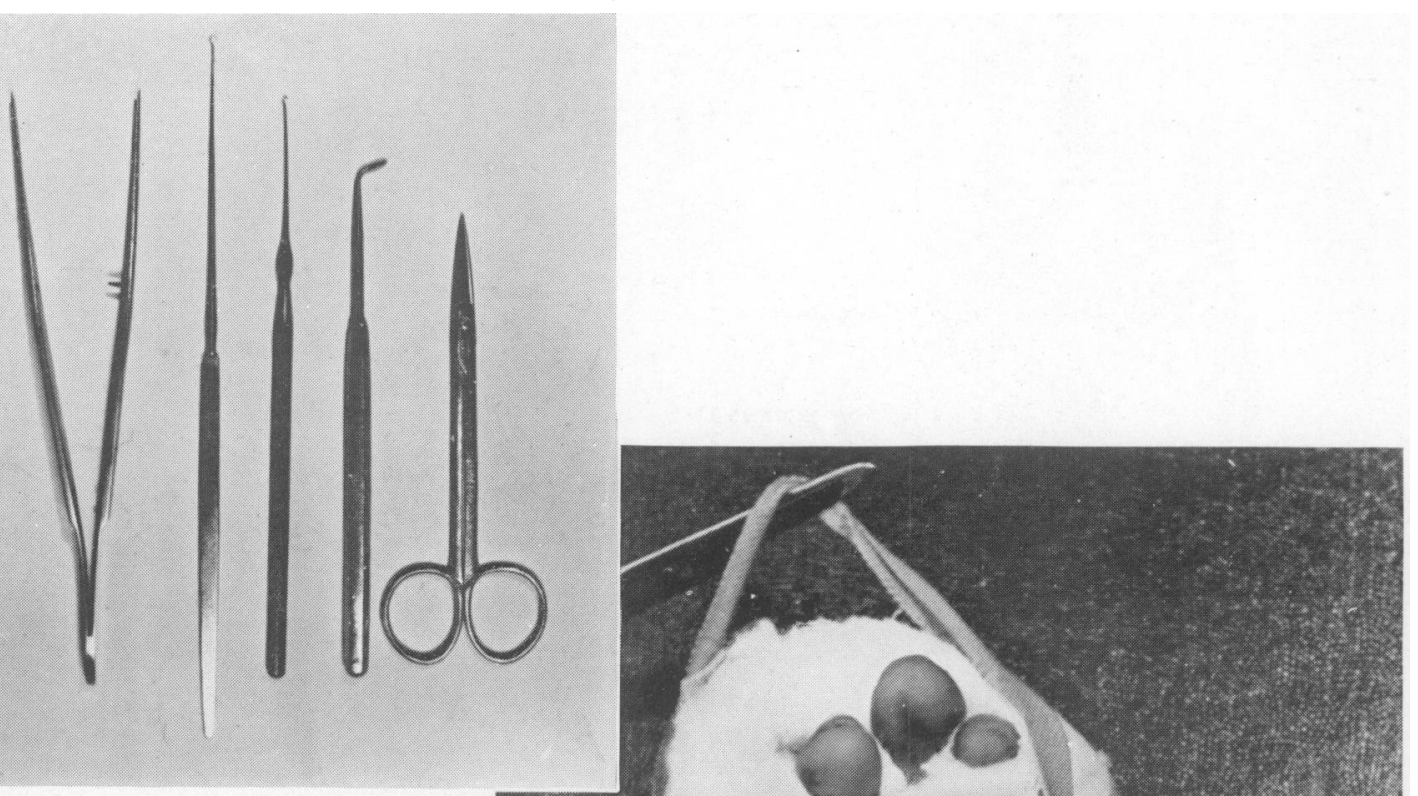

FIG. r.-Fine instruments used in hand dissection.

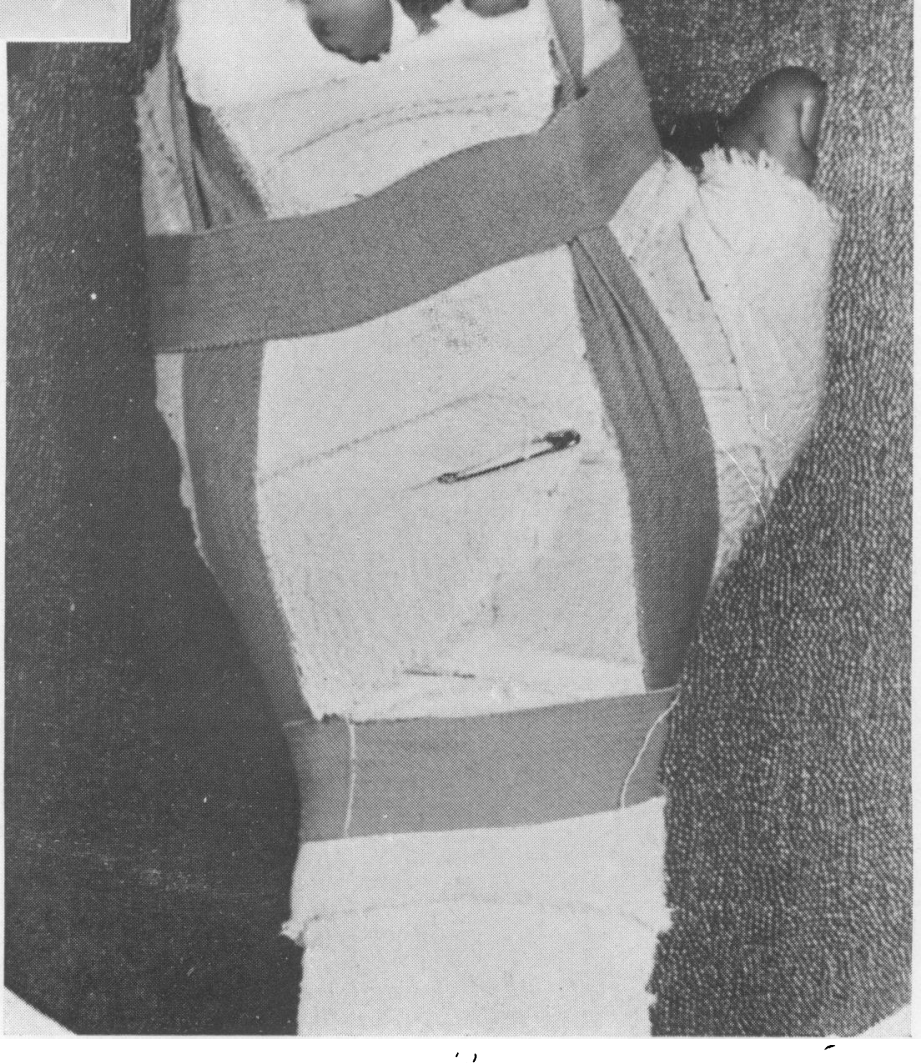

FIG. 2.-Pressure dressing of wool, crepe bandage and strapping. 

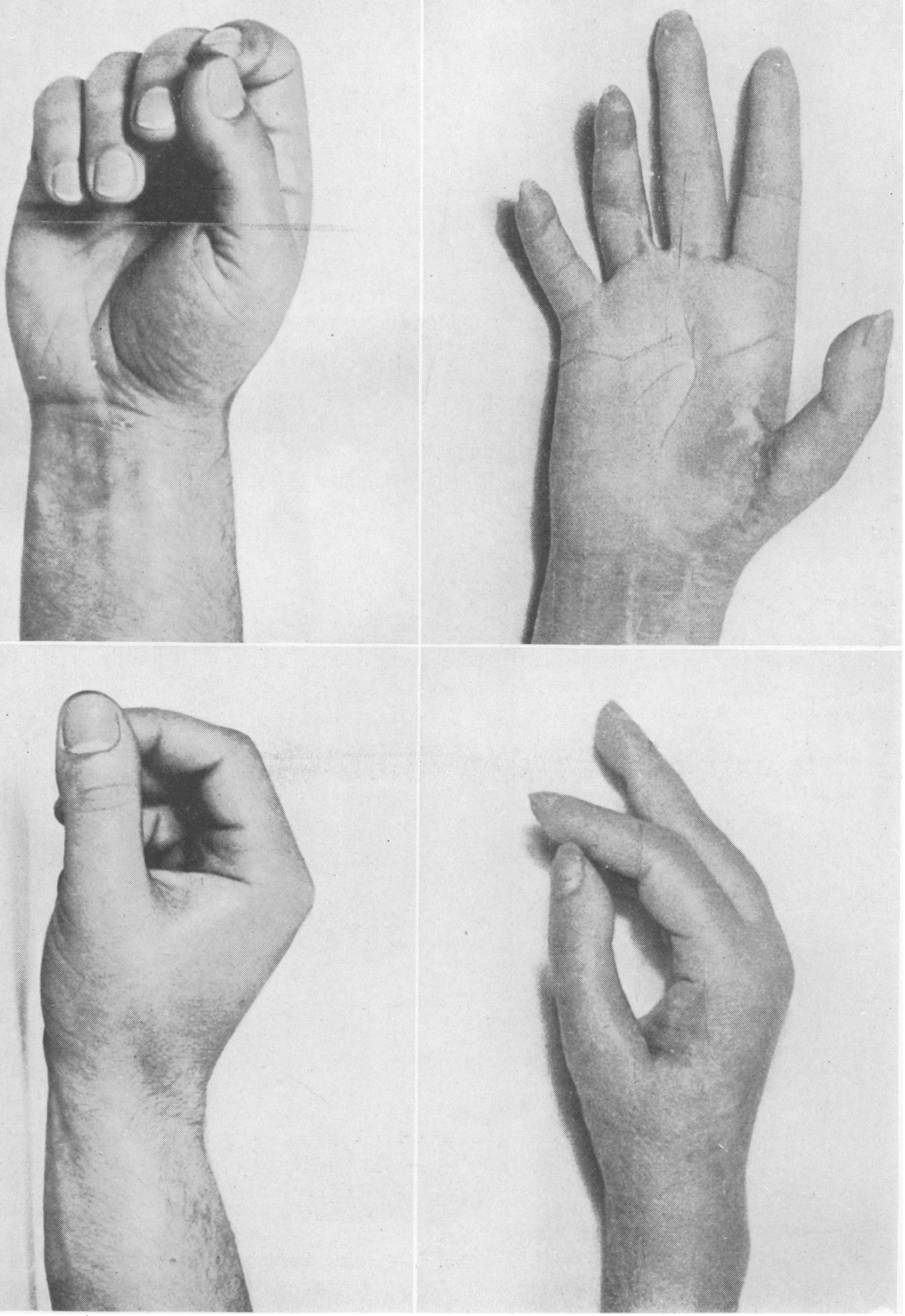

Fig. 4.-The position of function. Fig. 4.-The position of function.

Fig. 3.-The frozen hand resulting from a crush injury. Fig. 3.-The frozen hand resulting from a crush injury. 

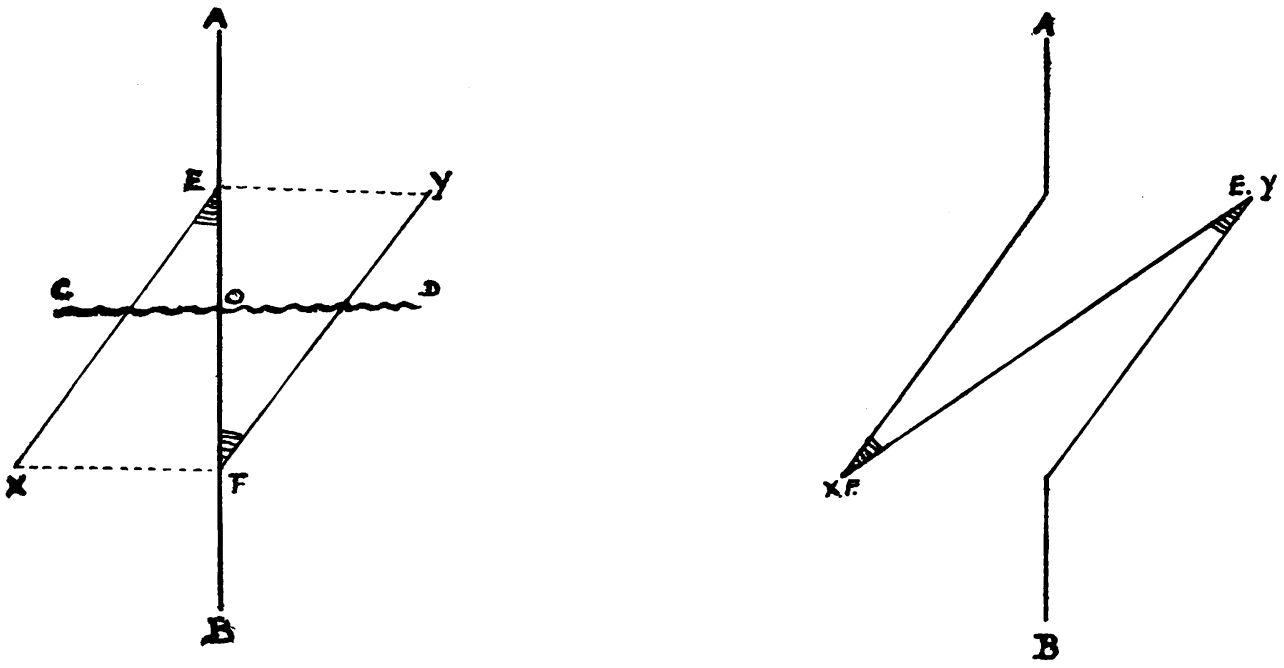

FIG. 5.- The Z plastic transposition of flaps.
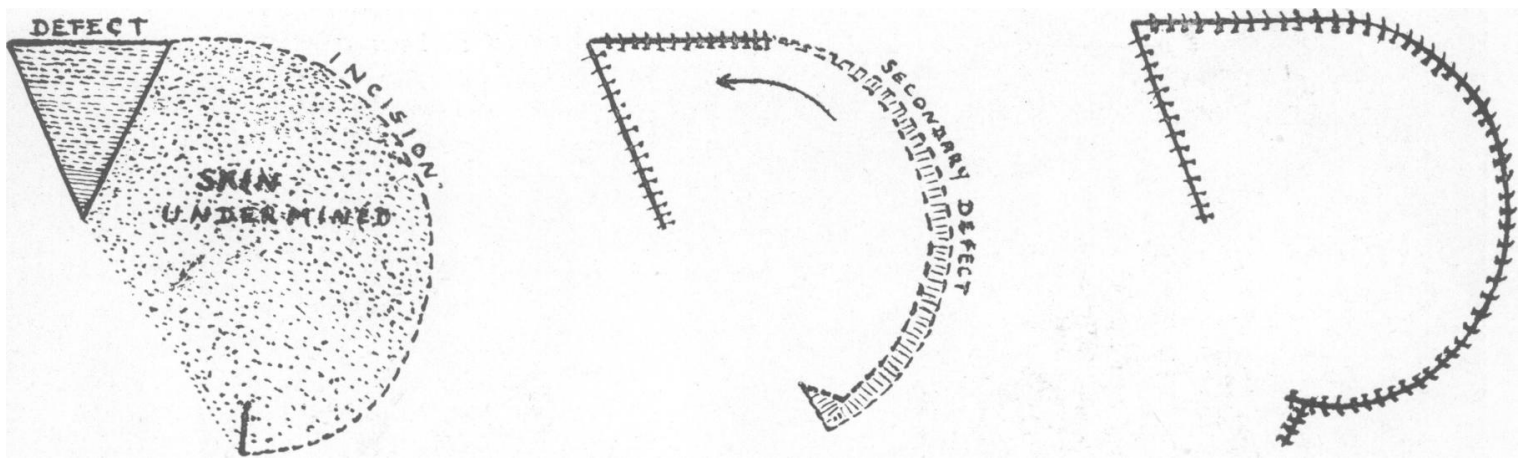

FIG. 6.-The Rotation Flap.
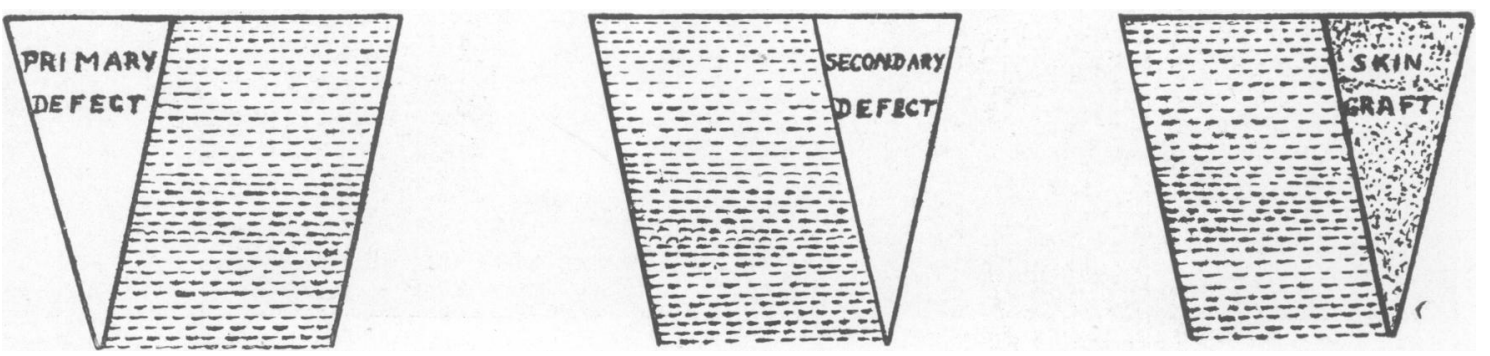

Fig. 7.- The Transposed Flap. 


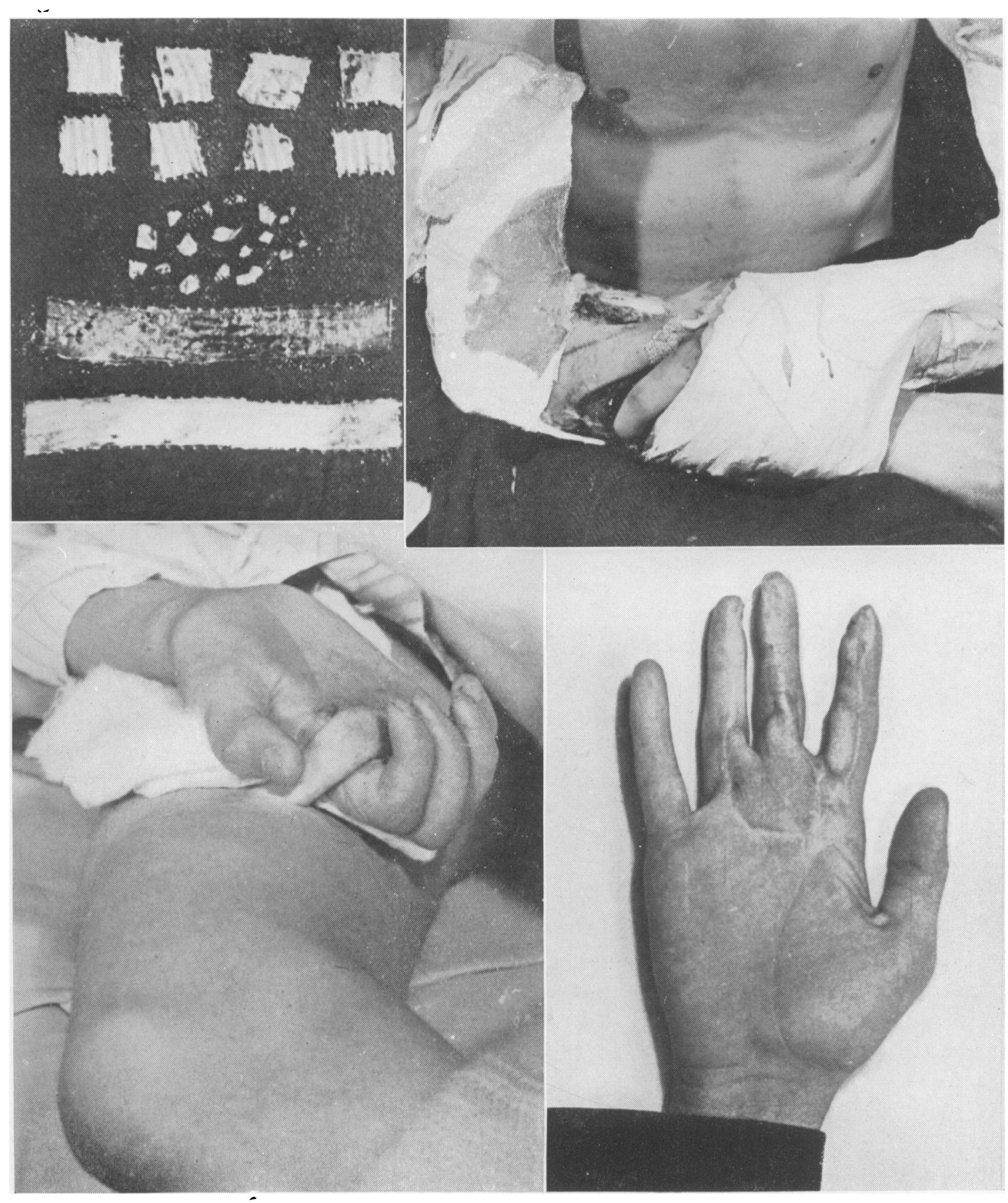

Fig. 8.-Strip grafts, spot grafts and patch grafts. FIG. 10.- Thigh flap for the repair of a palmar defect in the region of the second and third webs.
FIG. 9.-Showing method of immobilizing a cross arm flap.

FIG. I I.-Thigh flap inset into hand. 


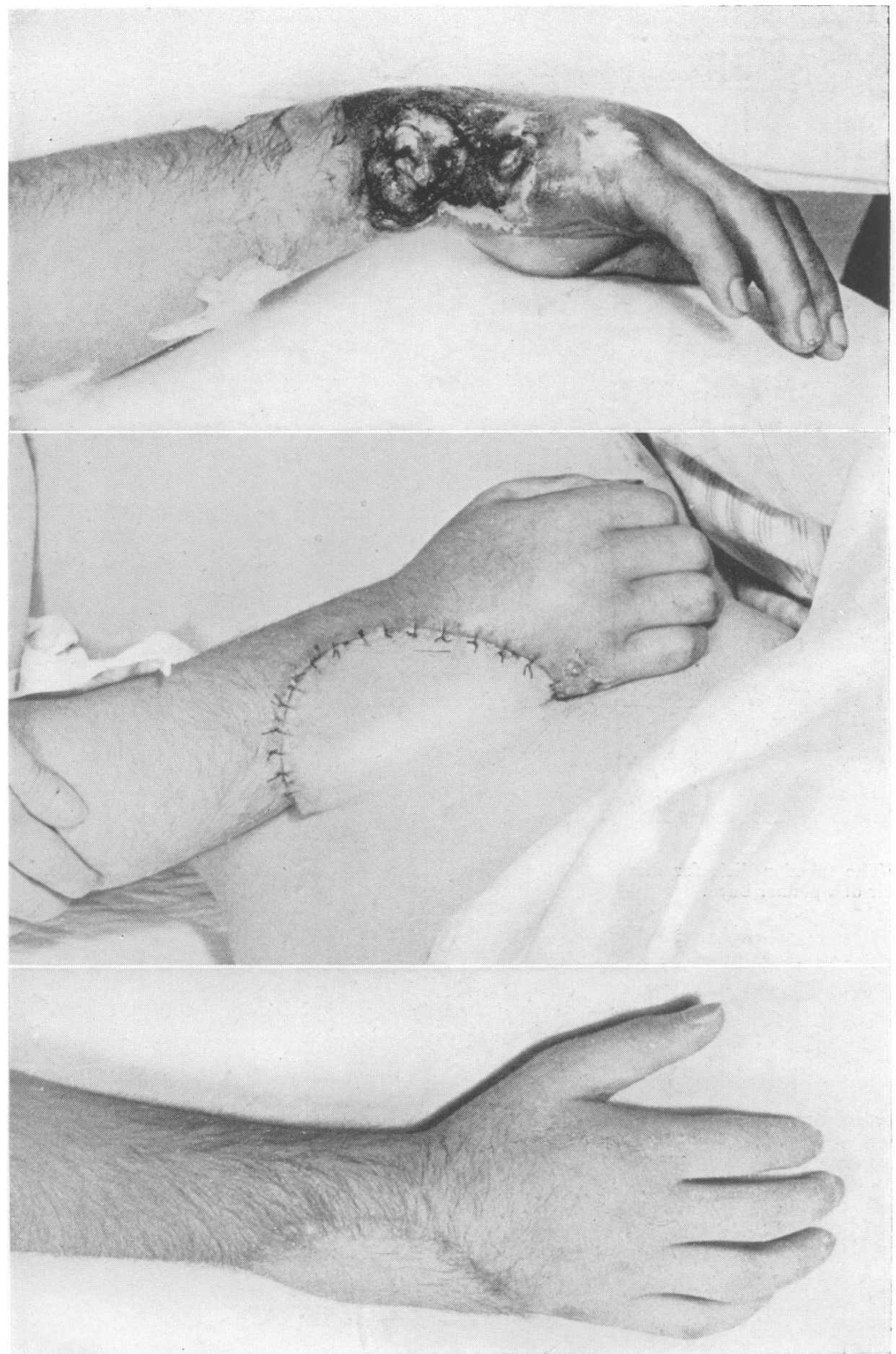

Fig. 12.-Abdominal flap repair for electrical burn of the ulnar border of the wrist and hand. The burn. FIG. I3.-The abdominal flap attached to the hand.

FIG. 1 1.- The inset completed. 


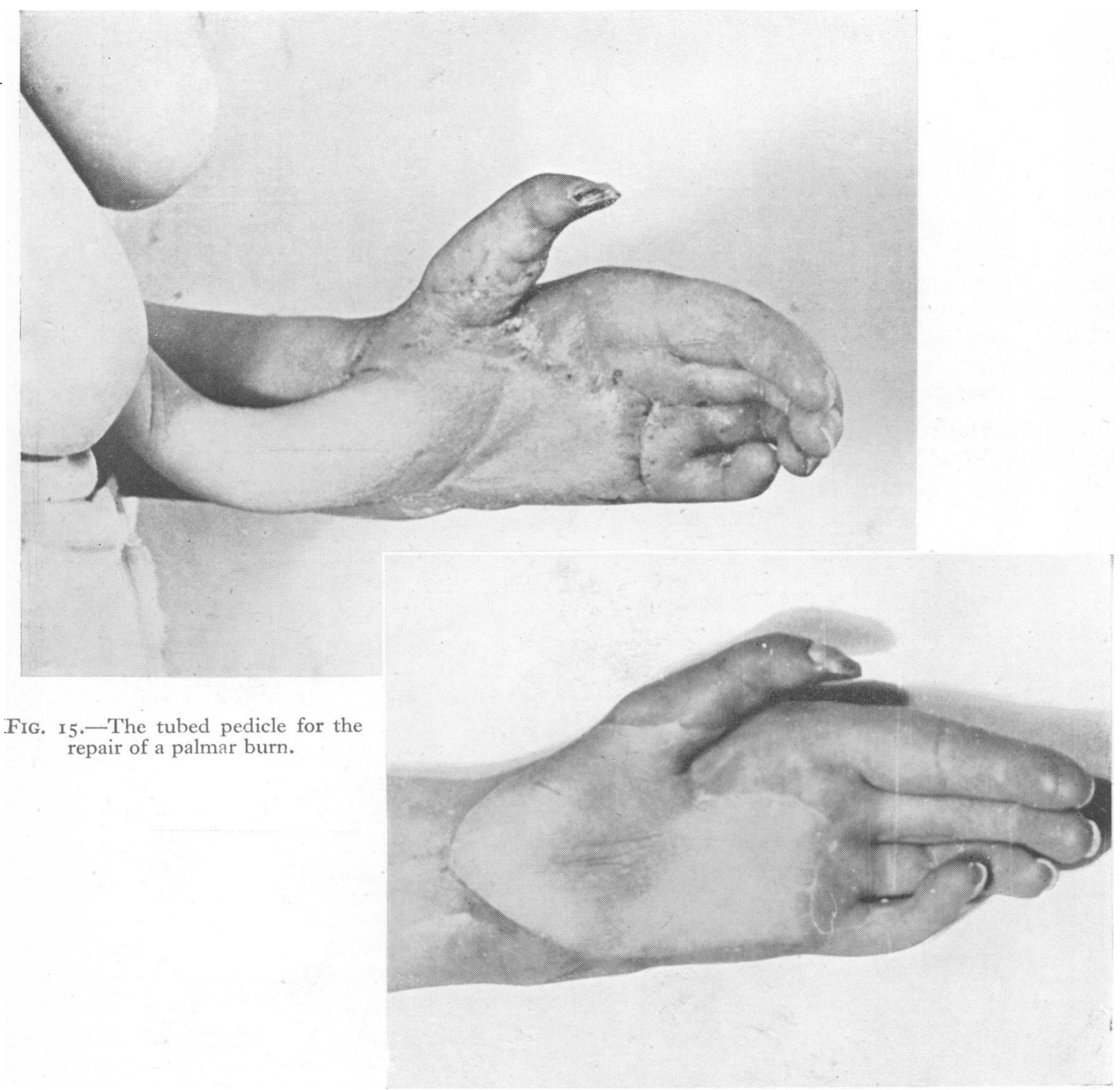

Fig. 16.-Inset of the pedicle completed. 
subcutaneous tissue plays a dual role. It acts as a cushion for the skin protecting it from trauma, and also guards the tendon and its sheath and the nerves and vessels against injury and subtraumatic stimuli which are constantly being applied to this surface.

On the dorsum of the fingers, the function of subcutaneous fat is of much less importance ; apart from its minor protective role, it conveys the venous plexus up to the hand.

Here on the hand, the fat layer, although normally thin, allows the free skin movement essential to flexion and extension of the fingers. This movement is nearly $\frac{3}{4}$ in. at the knuckles between the extremes of the range at the joints. It also allows unfettered movements of the extensor tendons and provides a medium through which the venous and lymphatic vessels course.

On the palm it is the great shock absorber. Intimately connected with the palmar fascia, it is movable and accommodating in flexion but tightly stretched in extension and is thus well adapted to the various functions of the hand.

\section{Dermis}

Through the dermis, the epidermis receives its nutrition and in it are found many of the sensory organs and nerve plexuses. The normal metabolism and stability of the skin depend upon an intact dermis and the instability of scars is often due to its absence or to defects in its structure.

On the volar surface of the hand and fingers where, owing to constant abrasion, the wastage rate of epithelium is high, these functions of the dermis are of great importance. On the dorsum of the fingers, however, satisfactory function of the skin is possible in the partial absence of the dermis and on the dorsum of the hand, this is also true provided the subcutaneous layer is intact.

From these considerations, we can examine the indications for replacement after injury.

On the volar surface of the fingers a full thickness skin and subcutaneous tissue loss should be replaced in all its three layers. In the palm, the central area between the thenar and hypothenar muscle groups again demands replacement of all three layers. A free skin graft over the muscles will, itself, be satisfactory because of the good blood supply and suppleness of the base.
On the dorsum of the fingers neither the subcutaneous tissue nor the whole thickness of the dermis need be replaced; and a split skin graft proves perfectly adequate in all cases. On the dorsum of the hand, provided there is a subcutaneous tissue covering over the tendons, a split skin graft will again be satisfactory. It is possible to do tendon surgery later through this split skin graft if it is really thick ( $\frac{3}{4}$ to $\frac{7}{8}$ skin thickness) and if the primary 'take' has been good.

When the dorsum of the hand has been denuded of its fatty layer, as well, all three layers should be replaced if free finger movement is to be expected.

\section{Methods of Repair of Tissue Loss}

\section{Direct suture}

This method is applicable only to the smallest of wounds and on the volar surface of the hand and fingers there is very little excess of skin available for the repair. Under no conditions should tension suturing be resorted to as the inevitable damage to the skin edges and the subsequent widening of the scar will reproduce the condition that the wound suture was designed to prevent. On the dorsum there is greater laxity of tissue, and by undermining the skin in the subcutaneous plane certain small defects may be closed. Transverse tension across the dorsum over the knuckles should be avoided as the full width of skin is required here for free flexion at the metacarpophalangeal joints.

\section{Local skin flaps}

There is a limited application for these methods and two are in common usage for small skin losses.

(a) Rotation flap. The rotation flap is useful for closure of triangular defects provided the flap incision does not cross flexion creases at right angles and so encourage secondary contractures. The main indication is for defects in the distal palm, the thenar eminence, or on the dorsum in the knuckle region. All rotation flaps on the hand should be based proximally so as to ensure venous drainage. Congestion and thrombosis are the penalties for distally placed flaps. The rotation flap is cut by extending the short side of the triangular defect by an incision which sweeps round the 
arc of the largest possible circle compatible with the presenting surface, and ideally should terminate at a point on the projection of a line drawn through the long axis of the triangle. The extension of the incision to this point is not always possible as the base of the flap is thereby narrowed under the blood supply and the skin is impoverished. In practice then the incision is lengthened until the flap, when undermined, will close the defect without tension. When properly designed, this flap should leave no secondary defect requiring closure by a free graft.

(b) Transposed flap. The principle of this method is to transpose the defect from one site to another where it can be suitably closed by a skin graft. Thus defects in the central palm can be transposed to the ulnar border of the hand where a split skin or Wolfe graft can be expected to take and stabilize without scarring and risk of subsequent breakdown. A central palmar defect exposing tendons and nerves can in such a manner be closed by incising across the palm to the ulnar border and then turning the incision proximally upwards towards the wrist. When the palmar skin is undermined the flap outlined will move across to close the primary defect, leaving a secondary triangular defect over the abductor minimi digiti which is a good base for the reception of a skin graft. This principle can be used in the treatment of defects in the interdigital webs and for small skin losses on the volar aspect of the fingers. In both these instances, the defects can be transposed to the sides or backs of the fingers, in which situations skin grafts are satisfactory.

\section{Free grafts}

The free skin grafts which were formerly described as either Thiersch grafts or Wolfe grafts, are now subdivided into a number of categories. In the hand there are important indications for adapting a particular type of graft to the area grafted, and much importance should be attached to this if optimum results are to be obtained.

The emergency surgery of hand defects offers great scope for the use of the free graft. It is by far the most useful method of repair and the saving of time and disability should recommend it to those who deal with acute hand injuries.

\section{Skin Grafts}

\section{THICK}

Wolfe Graft Full thickness .045 in to .032 in.

Pinch Graft .045 in. at centre .008 in. at periphery.

\section{THIN}

Half skin thickness .020 in.

One-third split skin .01 2 in. $\left\{\begin{array}{l}\text { Spot gratts } \\ \text { Patch ", } \\ \text { Strip ", }\end{array}\right.$

Thiersch Graft .008 in. to .0ro in.

\section{Wolfe Graft}

This graft includes the whole of the dermal and epidermal layers. It is cut either freehand or in large areas with a Padgett dermatome. Of all skin grafts it is the least ready to take and requires every refinement of technique to ensure successful transplantation. The common donor sites are the abdomen and inner aspect of the upper arm. The donor site must be closed by suture or by a split skin graft to avoid prolonged healing and unnecessary scarring.

If cut freehand, a pattern of the defect is marked on the donor skin and outlined with a knife, one edge is lifted with a skin hook and by careful dissection the graft is split off between the dermis and the subcutaneous fat. Not a vestige of fat should remain on the graft or the take will be jeopardized. The skin must be handled without trauma and should be manipulated only with the fingers or with a sharp hook. The crushing effect of forceps will cause points of necrosis in the devitalized skin.

To prevent collections of serum or blood under the graft, it should be punctured at halfinch intervals over its whole surface with a large, straight cutting needle or with a finepointed knife. These puncture wounds heal rapidly after allowing drainage from the wound in the first crucial hours after operation. Graft drainage has an important place in the hand where irregular surfaces are to be covered and absolute immobility is difficult to obtain.

The Wolfe graft is accurately sewn into the edges of the defect by either interrupted fine silk sutures or by a running mattress stitch. Exact edge to edge alignment and eversion are necessary if marginal scarring is to be reduced 
to a minimum. As with all free grafts, a firm pressure dressing and immobilizing splint is used during the first seven to ten post-operative days, after which time the dressing is removed and the graft inspected. Further care consists of protective dressings, general exercises and measures to encourage stability of the skin.

Infection or the risk of infection is an absolute contraindication for the use of a Wolfe graft. One should hesitate to employ it on a hand injury more than six to eight hours old, and only then after a careful debridment has been done, particularly if there has been a 'crush' element present.

The principle indication for the Wolfe graft is for the replacement of skin defects of the volar surfaces of the fingers and thumb in which the subcutaneous layer is intact. Here the full buffering effect of the dermis is important and this layer should be replaced when destroyed. Whatever the shape or size of the defect, if it crosses a flexion crease on a digit it should be enlarged until its lateral margins run along the lateral axes of the finger, or a triangle of intact skin should be sacrificed with its apex at a point on, or posterior to, this line. In this way, a secondary contraction of the marginal scar can be prevented.

The lateral axial line is determined by flexing all the finger joints and viewing the digit from the side. The posterior limits of the flexion creases are marked and when the finger is straightened a line is drawn through these points. It will be noted that this line is a little more posterior than would at first be apparent.

In practice any longitudinal incision anterior to this line may, during healing, cause a flexion contracture, so that incisions for access to the finger should be designed accordingly.

A common indication for the Wolfe graft is for the repair of skin losses on the finger tips. An accurate diagnosis of the loss should be made and if there has been destruction of much of the pulp substance this must be replaced by other methods.

A common tip injury is caused by a bread or bacon slicer which removes a circular area of skin off the terminal pulp. This defect has sloping margins, the loss being thickest in the centre and when small may confidently be repaired with a Pinch graft. The skin for this graft is elevated on a needle point and while tented upwards is sliced off cleanly with a knife. It is thin at the edges and full thickness in the centre, and is well suited to these small defects.

Where possible the proximal edge of tip defects should be trimmed to run parallel with the papillary ridges in this region then the scar will be minimal and there will be less likelihood of secondary pulp distortion.

The Wolfe graft is also indicated for closure of central palmar defects in the triangle bounded by the bases of the fingers and the borders of the thenar and hypothenar eminences. Provided the subcutaneous layer is intact, these grafts do well and may even be satisfactory over small exposures of palmar fascia. Ragged wounds of the palm which need grafting are better trimmed into a triangular shape with the edges parallel to the various creases. Subsequent contraction of the marginal scars is reduced to a minimum, scars which if badly placed so easily cause deformities at the metacarpophalangeal joints and transverse palmar shortening.

\section{The Thick Split Skin Graft}

This graft, although differing little in thickness from the Wolfe graft, has certain different properties. It takes more readily on transplantation but the epithelium does not mature in quite the same fashion presumably owing to partial lack of dermal support. It is thus inherently slightly less stable than its full thickness counterpart. This minor defect can, however, be overcome by using it on an ideal base, and in the hand such an area is found on the thenar or hypothenar muscles. When there is skin loss in either of these regions or in the ulnar border of the first web which is formed by the first dorsal interosseus muscle, satisfactory and permanent function of the graft can be expected. In any appreciable size the thick split skin graft can be cut only with a dermatome, a pattern should be used and its method of application is the same as for a full thickness graft.

\section{The Thin Split Skin Graft}

This is the graft used to replace the skin on the dorsum of the hand and fingers. Normally the dorsal skin is much thinner than that on 
the volar surface and its function is that of an elastic rather than a protective covering or an organ of touch. Thermal stimuli are more readily appreciated through the dorsal skin, but it lacks fine discriminatory sensibility which is found on the palmar aspect of the fingers.

These grafts take more easily than is the case with thick grafts, and can even be relied upon, if drained by multiple punctures, to survive in the presence of infection. Demanding less nutriment for survival they can be based on relatively avascular capsular or tendon tissues and are of great value in the early treatment of burns. The one-third thickness graft provides an adequate permanent cover for the dorsum of the fingers, and half thickness skin is used for the knuckle region and the dorsum of the hand. The tendency for the thinner graft to contract after healing is compensated for by the pull of the long flexor tendons and by the absence of concave surfaces on the dorsum. Provided there is a good initial take subsequent contractures should not arise.

\section{The Spot, Patch and Strip Grafts}

The grafts previously described are used in sheets. Each sheet covers completely an area denuded of skin. Even for the thinnest sheet graft; infection, with its liberation of pus and exudate from the wound surface, is a formidable deterrent to adhesion. A relatively small amount of exudate will balloon the graft off its bed and the regenerative processes between the two become impossible. The accumulation of toxic products macerates both the skin and the wound surface, so that by injudicious grafting the healing time may be greatly prolonged.

Various advantages are gained by dividing thin skin up into small units, and the three in common use are spots (the size of pin heads), patches (from $\frac{1}{2}$ to $1 \mathrm{~cm}$. square), and strips $\left(\frac{1}{2}\right.$ to $1 \mathrm{~cm}$. in width and variable in length). These units are placed on the wound surface leaving spaces between them so that drainage can escape into the dressings and so be conducted away from the deep surface of the skin. The problems of movement are not so acute with this method as motion at a joint is not necessarily transmitted over the whole of the grafted skin. Further, for a given amount of skin cut from a donor site a much larger area can be covered which, although it has little application in hand surgery, may be of considerable importance in widespread burns.

In general, the heavier the infection the smaller the graft unit used. Thus even when there is frank pus on a recent wound it is possible to obtain a good take by spot grafting. Patches and strips are used on less heavily infected surfaces, but in the presence of a good blood supply remarkable results can be achieved by their use, even in the presence of a fairly profuse infection.

These graft units coalesce by epithelial regeneration from their edges, thus the intervening areas are held by scar epithelium. Graft units should therefore be placed close together leaving just a millimetre or less for drainage between them, and strips should be placed in the long axis of the dorsum of the hand, transversely on the volar surface of the fingers and parallel with the normal creases on the palm. This prevents the development of scars which are at variance with the principles of surgery of the hand, early freedom of movement is possible, secondary deformities are less likely to be troublesome. So great is the importance of early epithelialization of hand wounds and burns, that with these methods at our disposal grafting procedures should not be left until the defects are sterile. Each day that a raw surface is allowed to remain detracts from the final result.

\section{Pedicle Grafts}

These more complicated methods of repair have a place in the immediate treatment of hand injuries. These procedures are done in two stages because the grafts contain skin and subcutaneous tissue and must thus be transplanted in vascular continuity to survive. The impossibility of transplanting skin and fat together as a free graft has been mentioned, bnt there are frequent occasions for the replacement of both these layers, and it is then that the pedicle method of transference from distance is used. Briefly, the indications for pedicle grafting are skin and subcutaneous tissue losses on the volar surface of the fingers, the central palmar triangle and the dorsum of the hand.

There are two contraindications for the use of this method. If the wound is more than 
six to eight hours old and, by the nature of its causation, infection is likely to occur, the pedicle flap should not be used. Similarly if the tissue loss, is caused by a severe crushing injury or is accompanied by a crush in another part of the hand, the difficulty of controlling oedema and the subsequent prolonged joint stiffness rule out this method as a primary repair. Under these conditions it is preferable to close the defect by one of the free grafts, whichever may be indicated, regarding this as a temporary skin dressing which can be removed when the danger of infection and oedema is passed and proceeding then with the pedicle flap reconstruction.

A suitably chosen free graft will overcome the problem of infection and a pressure dressing and elevation will control the oedema.

There is some evidence to show that treatment by cooling may be effective in the prevention of oedema if ice packs are used postoperatively. The full pressure dressing and elevation routine may be discarded and the use of primary flap repairs may be extended to certain crush injuries.

The variations in this type of transplantation are limitless and much depends upon the ingenuity of the operator as to the method of choice for a given defect. The ideal is to replace lost tissues by similar tissues both in quality and in quantity. Thus it is ridiculous to replace the dorsum of a hand with an epidermal flap containing the full thickness of the abdominal fat, the result is functionally and cosmetically grotesque, nor is it reasonable to replace digital skin with a bulky mobile flap which on compression flows aimlessly round the supporting phalanges. Accurate quantitative replacement is just as important as the proper choice of the donor site.

\section{Examples of Pedicle Flaps}

\section{The repair of central palmar defects}

The commonly advocated abdominal flaps are not ideally suited to this type of case. In order to prevent the flap from kinking in the bridge between the abdomen and the hand, the latter must be held in full supination. This is an unnatural position and is impossible to maintain unless a massive plaster jacket with an arm extension is made. If pronation does occur the contact between palmar skin and the abdomen encourages sweating and maceration of the skin and infection of the suture line is difficult to prevent.

The best choice lies between the opposite forearm or the internal aspect of the thigh on the same side as the donor site and of the two, the forearm is usually more convenient.

A suitable position for the flap is found on the front of the forearm and is based proximally. The flap is raised and the donor defect grafted, using a $\frac{3}{4}$ split skin graft. The flap is sewn into the palmar defect and the two arms are immobilized together in plaster. The position is fairly comfortable and easy to maintain for two weeks when severance of the flap is completed.

The same technique is used for the thigh flap but here the problems of immobilization are much more difficult and it is better to reserve the operation for children and adolescents in whom such fixation is less irksome.

\section{Abdominal flap}

This flap should be restricted to the repair of defects on the dorsum of the hand. The position during transfer is natural and ample material is available. The flap can be based above and medially, below and laterally, or can have a double base and thus become "a bridge flap.' The donor site is the lower quadrant and should be somewhat more lateral in women owing to the excessive fatty deposition over the lower third of the rectus muscle.

When an abdominal flap with a single base is contemplated, it should be designed so that the base is at least equal to the length of the flap. The circulation will then be sufficient. When this rule is followed it is permissible to trim off all excess of fat and leave just enough in the flap to replace the subcutaneous tissue loss on the hand. It is sometimes necessary to enlarge the hand defect so that a safe flap of this type can be used and there need be no hesitation in so doing.

When there is a narrow defect crossing the entire width of the dorsum a bridge flap can be used and a safe ratio between length and base in this case is 5 to 2 .

The donor site of the flap should be closed by a split skin graft so that all raw areas are covered. Failure to obtain complete closure is the commonest cause of sepsis, delayed healing, and scar formation in the flap. 
When the flap has been sewn into the defect, the position of the hand and arm is maintained by strapping it to the chest and abdomen with elastoplast. With children, however, it is safer to use plaster immobilization and for them a light plaster jacket with a rigid arm extension serves the purpose well.

The second stage is done within two or three weeks. The base of the flap is severed and set into the margin of the hand defect. This should truly be a marginal inset as in all cases at least $\frac{7}{8}$ ths of the flap should be attached to the hand at the first operation, the design allowing for just a short bridge between the abdomen and the hand.

These flaps allow successful extensor tendon suture to be done during the first stage so long as the fingers can be splinted in full extension. They also provide an excellent medium for subsequent tendon grafting, and the grafts can be tunnelled through the fatty layer underneath the flap.

\section{The Tubed Pedicle}

This cannot be used in an emergency as a means of importing tissue as the skin tube must be formed on the abdomen or chest at least three weeks before transference can be made. In gross injuries, however, there are occasions when a temporary skin graft should be used for the hand and subsequently replaced with skin and fat. In these cases much time and an operative stage can be saved if at the emergency operation a tubed pedicle is made which will be ready to transfer to the hand after the acute phase of the injury has subsided. The planning of tubed pedicle repairs is fraught with difficulty, and an accurate forecast of the whole surgical programme must be made before the size and situation of the tube is decided upon. An error in judgment at the first operation may jeopardize the whole result and it may not be apparent until several subsequent stages have been completed, by which time the mistake may well be irrevocable.

\section{Conclusions}

The pathology and treatment of recent skin and subcutaneous tissue injuries of the hand are viewed from the angle of the plastic surgeon. Some of the more general aspects of the burnt hand are mentioned but in view of the extensive literature already in existence, details are not given. The basis of early treatment of these injuries is the prevention of infection, oedema and granulating surfaces.

\section{BIBLIOGRAPHY}

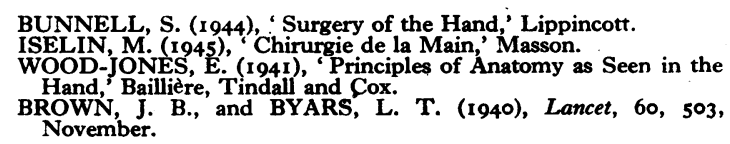

\section{EDITORIAL (continued from page 452)}

In cases of extrahepatic block, where the portal vein itself is affected, porto-venocaval anastomosis is not feasible and spleno-renal anastomosis has to be carried out. Technically, Blakemore and Whipple find the vitallium tube technique to be of great value but a tube of adequate calibre should be used. Failure from thrombosis at the site of anastomosis has been uncommon ; it should be noted, however, that successful anastomosis is more likely to be obtained in cases of portal hypertension than in ordinary vein-to-vein anastomosis, where failure is common as a result of the low pressures in the systemic veins. In portal hypertension the pressure in the portal vein is high, and there is a considerable difference between the pressure in it and in the vena cava. Success in these cases for this reason should approach the success commonly obtained in arterial anastomosis.

Seventeen of Blakemore's cases have been followed up for more than six months and some up to two years. In addition to the disappearance of haemorrhages and ascites the majority have shown considerable gains in health, weight and appetite and improvement in liver function.

\section{BIBLIOGRAPHY}

Whipple, A. O., Annals of Surgery, 122, Oct., 1945. Blakemore, A. H., Annals of Surgery, 122, Oct., 1945. Blakemore, A. H., S.Y.O., 84, 645, April, 1944. 